\title{
ANÁLISE DE SENSIBILIDADE DE TÉCNICAS APLICÁVEIS PARA A REDUÇÃO DO CONJUNTO DE TREINAMENTO E TESTE DE REDES NEURAIS ARTIFICIAIS
}

\author{
João Ricardo S. de Souza*. Raphael Batista** \\ *Departamento de Eletroeletrônica e Computação, CEFET-MG Campus Contagem, \\ Alameda das Perdizes, $n^{\circ}$ 61, 32146-054, Contagem, Minas Gerais, Brasil. (e-mail: \\ joaor@cefetmg.br) \\ **Programa de Pós-Graduação em Engenharia Elétrica - Universidade Federal de Minas Gerais, \\ Av. Antônio Carlos, $n^{\circ}$ 6627, 31270-901, Belo Horizonte, Minas Gerais, Brasil (e-mail: \\ raphaelbatista@ufmg.br)
}

\begin{abstract}
During the application of Artificial Neural Networks for solving classification problems, sometimes the user faces extremely large datasets. This makes the procedure for the training, testing and validation of this Neural Network to become computationally costly. There are several techniques that can be used to reduce the size of this training dataset. In this work, a sensitivity analysis of four of these techniques will be presented, where the reduction obtained in each one of them and the impact of their use on the accuracy of Artificial Neural Networks will be verified. For this action, two synthetic classes are developed from normal probability density functions, with the change of their respective variances, to evaluate the impact of superposition between classes. In addition, the amount of standards is also varied in this work.
\end{abstract}

\begin{abstract}
Resumo: Durante a aplicação de Redes Neurais Artificiais na solução de problemas de classificação, por vezes o usuário depara-se com conjuntos de dados extremamente grandes. Isso faz com que o procedimento de treinamento, teste e validação desta rede neural se torne computacionalmente custoso. Existem, na literatura, diversas técnicas que podem ser utilizadas para reduzir o tamanho deste conjunto de treinamento. Neste trabalho, será apresentada uma análise de sensibilidade de quatro destas técnicas, onde será verificada a redução obtida em cada uma delas e o impacto de seus usos na acurácia da rede neural artificial. Para tal ação, duas classes sintéticas são desenvolvidas a partir de funções de densidade de probabilidade normais, com a alteração de suas respectivas variâncias, para avaliar o impacto da superposição entre as classes. Além disso, a quantidade de padrões também é variada neste trabalho.
\end{abstract}

Keywords: Artificial Neural Networks; Gabriel graph; Condensed Nearest Neighbor; Support Vector Machine; Edge Recognition Algorithm.

Palavras-chaves: Redes Neurais Artificiais; Grafo de Gabriel; Condensed Nearest Neighbor; Máquina de Vetor de Suporte; Algoritmo de Reconhecimento de Bordas.

\section{INTRODUÇÃO}

Usuários de redes neurais artificiais (RNA) eventualmente precisam tratar conjuntos de dados muito extensos. Isso pode ocorrer em casos em que os dados são gerados aleatoriamente por meio de simulações computacionais que utilizem o Método de Monte Carlo, como nas aplicações relacionadas à proteção de sistemas elétricos de potência (Perez, 1994; Dalstain e Kulicke, 1995; Sidhu, Singh e Sachdev, 1995; Souza, Pereira e De Conti, 2017). Em tais situações, para que o conjunto de dados utilizado para o treinamento da RNA possa ser considerado suficientemente representativo da realidade, é necessário que sejam feitos um número elevado de simulações. Além disso, uma vez que este tipo de aplicação geralmente trabalha com sinais de tensão e corrente variáveis no tempo, os dados de entrada da RNA são oriundos de janelas móveis destas fontes, seja utilizando diretamente as amostras da janela ou alguma grandeza calculada a partir destas, como os fasores fundamentais estimados. Desta maneira, cada um dos casos simulados gera uma quantidade significativa de dados, o que acrescenta novos dados ao conjunto de treinamento, já considerado grande.

A adoção direta de um conjunto de dados grande torna o processo de treinamento, teste e validação caro do ponto de vista computacional. Assim, um dos principais desafios para o uso de RNA em aplicações desta natureza é a diminuição do conjunto de treinamento por outro, dito subconjunto consistente mínimo do original.

Há diversas técnicas que podem ser empregadas para reduzir o conjunto de dados do treinamento, embora seu uso desvie 
de suas propostas originais, caso da identificação dos vetores de suporte e o balanceamento de classes. A redução do conjunto de treinamento se torna uma consequência indireta da aplicação destes métodos.

Neste trabalho, quatro diferentes metodologias são tratadas para tal fim. A primeira se baseia no Grafo de Gabriel (Gabriel e Sokal, 1969; Zhang e King, 2002), enquanto a segunda é a CNN (Condensed Nearest Neighbours), descrita em (Hart, 1968; Lacerda, 2006). A SVM-KM (Support Vector Machine with k-means), tratada em (Barros de Almeida, Braga e Braga 2000) e, por fim, uma simplificação do algoritmo de reconhecimento de bordas (Edge Recognition Algorithm - ERA) (Ahmadi e Ghaffari, 2014) são considerados neste texto.

Para analisar a sensibilidade das técnicas mencionadas, a redução média do conjunto de dados proporcionada por cada uma delas e o impacto delas na acurácia da RNA foram avaliadas. Dois conjuntos de dados foram sintetizados com o objetivo de corresponderem a duas classes, compostos por 100 elementos cada um e desenvolvidos por meio do uso de funções de densidade de probabilidade normais. A variância e a quantidade de padrões são variáveis nesta situação.

Para a avaliação da redução da acurácia provocada pela a adoção destas técnicas, o desempenho de uma RNA do tipo Multilayer Perceptron (MLP), com três neurônios na camada escondida, foi estimado por meio do simulador de redes neurais Stuttgart (RSNNS), desenvolvido em linguagem $\mathrm{R}$ (Bergmeir e Benitez, 2012). Tal RNA, utilizada como classificador, é treinada com o conjunto de treinamento completo e com seus subconjuntos resultantes da aplicação dos métodos mencionados. A acurácia em cada um dos casos é calculada por meio da validação cruzada com quatro subconjuntos.

Este artigo está organizado como se segue. A Seção 2 apresenta as técnicas utilizadas para se fazer a redução no conjunto de dados testadas ao longo do trabalho. A Seção 3 discorre sobre os resultados obtidos e a Seção 4 apresenta as conclusões.

\section{METODOLOGIAS PARA A REDUÇÃO DO CONJUNTO DE TREINAMENTO}

O desenvolvimento de subconjuntos consistentemente mínimos por meio de conjuntos de dados requer algum cuidado. Como indicado em (Hart, 1968), se o risco bayesiano for baixo, ou seja, a superposição entre as classes for pequena, a tendência é que o subconjunto tenha somente os pontos que estão próximos à fronteira entre as diferentes classes.

Tal característica é importante na avaliação das técnicas vistas neste trabalho, as quais são apresentadas a seguir.

\subsection{Procedimento que utiliza o Grafo de Gabriel}

A primeira metodologia a ser testada utiliza o chamado Grafo de Gabriel. Grafos são conjuntos de arestas que interligam os pontos que compõem um determinado conjunto, sendo denominados planares quando tais segmentos de reta não se interceptam. Grafos de Gabriel são um tipo de grafo planar construídos por meio de hiperesferas, cujo diâmetro é igual às arestas. Neste tipo de grafo, uma aresta só é considerada válida se ela não contiver nenhum ponto (Gabriel e Sokal, 1969; Zhang e King, 2002). Em outras palavras, o segmento de reta que interliga os pontos $x_{i}$ e $x_{j}$ somente é válido se:

$\delta^{2}\left(x_{i}, x_{j}\right)=\delta^{2}\left(x_{i}, x_{k}\right)+\delta^{2}\left(x_{j}, x_{k}\right)$

para todos os pontos $x_{k}$ possíveis, em que $\delta(\backslash a, b)$ é a distância euclidiana entre os pontos $a$ e $b$ no espaço.

A Fig. 1 ilustra um conjunto de pontos em um espaço bidimensional que exemplifica o processo de construção de um grafo de Gabriel (Souza, Pereira e De Conti, 2018). Nesta situação, as hiperesferas correspondem a circunferências. Assim, o segmento de reta entre $x_{b}$ e $x_{c}$ corresponde a uma aresta válida porque a circunferência, cujo diâmetro é dado por $\left(x_{b}-x_{c}\right)$, não contém nenhum outro ponto. O segmento de reta que interliga os pontos $x_{a}$ e $x_{b}$, por sua vez, não corresponde a uma aresta válida porque a circunferência de diâmetro $\left(x_{a}-x_{b}\right)$ contém um outro ponto.

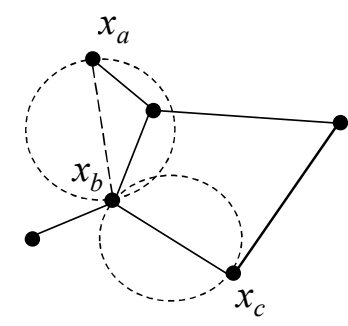

Fig. 1 Construção de um grafo de Gabriel.

A metodologia utilizada para reduzir o conjunto de treinamento utilizada neste trabalho é composta dos seguintes passos, ilustrados na Fig. 2:

1. Constrói-se um Grafo de Gabriel por meio dos pontos que compõem o conjunto de treinamento, vide Fig. 2 (a);

2. Retém-se somente as arestas compostas por pontos que pertencem às classes diferentes, conforme a Fig. 2 (b);

3. Retém-se apenas os pontos que formam tais arestas retidas no passo 2, como indicado na Fig. 2 (c).

\subsection{Procedimento que adota o $C N N$}

A metodologia CNN se baseia na regra do vizinho mais próximo (Hart, 1968; Lacerda, 2006). Esta regra enuncia que, caso haja um conjunto de amostras previamente classificadas e uma outra amostra a que se deseja classificar, a probabilidade maior é de que tal amostra pertença à mesma classe daquela a qual ela está mais próxima.

Para a aplicação desta regra, são desenvolvidos dois conjuntos, denominados STORE e GRABBAG, que são inicialmente vazios. Esta metodologia é composta dos seguintes passos: 


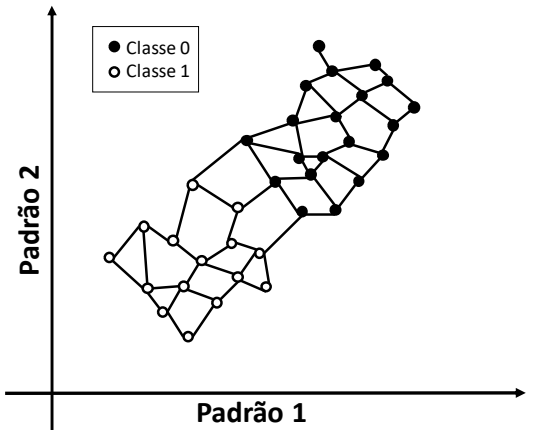

(a)

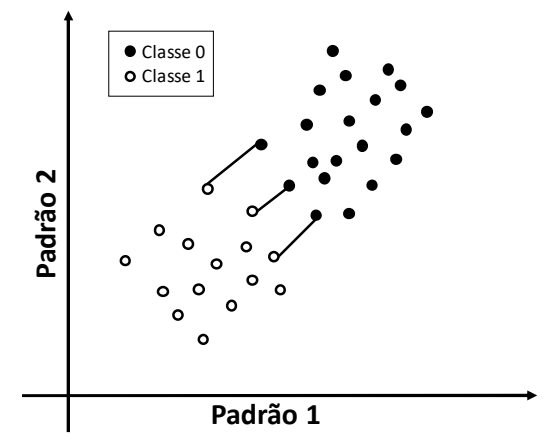

(b)

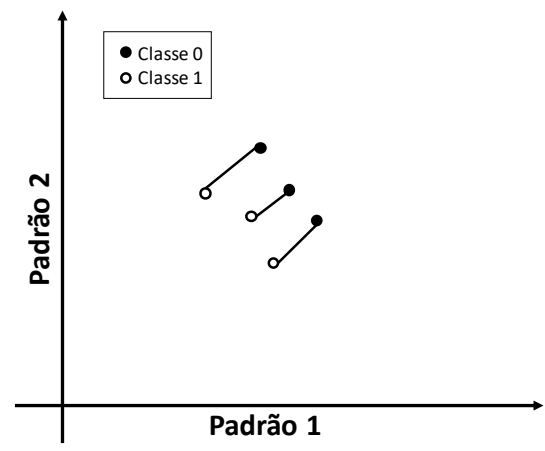

(c)

Fig. 2 Passos da metodologia proposta para a redução do conjunto de treinamento

1. Sorteia-se uma primeira amostra, a qual é armazenada no conjunto STORE;

2. Sorteia-se uma segunda amostra, classificada por meio da regra do vizinho mais próximo e utilizando o conjunto STORE como referência;

3. Se a classificação obtida no passo anterior for correta, esta amostra é armazenada no conjunto GRABBAG e, caso contrário, ela é armazenada no conjunto STORE.

4. Repetem-se os passos 2 e 3 até que todas as amostras do conjunto de treinamento original tenham sido percorridas.

5. Aplica-se novamente os passos 2 e 3 aos elementos do conjunto GRABBAG.
6. Repete-se o passo 5 até que o conjunto GRABBAG esteja esgotado ou que não haja modificação em duas iterações consecutivas.

Após o final da execução de todos estes passos, o conjunto STORE é utilizado como o conjunto de treinamento reduzido (subconjunto consistente mínimo).

\subsection{Procedimento que considera o SVM-KM}

A metodologia SVM-KM é utilizada para acelerar o treinamento de SVM (Barros de Almeida, Braga e Braga, 2000) e se baseia no método de treinamento não supervisionado k-means, que agrupa as amostras em k agrupamentos, em que $\mathrm{k}$ é um número definido pelo usuário (Hastie, Tibshirani e Friedman, 2009). O uso de tal técnica tem o seguinte roteiro:

1. Define-se um número $\mathrm{K}$ de agrupamentos (clusters), que serão definidos por meio da aplicação do algoritmo kmeans no conjunto de treinamento original;

2. Os dados dos agrupamentos que possuírem dados somente de uma classe serão removidos e somente seus centroides são mantidos no conjunto de treinamento;

3. Por outro lado, os dados dos agrupamentos que possuírem dados de mais de uma classe são mantidos no conjunto de treinamento.

$\mathrm{O}$ número de agrupamentos $\mathrm{K}$ ótimo a ser utilizado depende da esparsidade dos dados. Neste trabalho foram testados três diferentes valores de K: 20, 40 e 100 .

\subsection{Procedimento com a adoção do ERA}

O ERA também se baseia na regra do vizinho. Um ponto é retido somente se entre os seus $\mathrm{N}$ vizinhos mais próximos estiver um ponto de outra classe (Ahmadi e Ghaffari, 2014). $\mathrm{N}$ é um número definido originalmente pelo usuário. Neste trabalho, utilizou-se $\mathrm{N}=5$.

\section{DISCUSSÃO DOS RESULTADOS}

O teste do desempenho das técnicas de redução reuniu dois conjuntos de dados sintéticos desenvolvidos por meio de funções de densidade de probabilidade normais, cada uma composta por 100 elementos correspondentes a duas classes, denominadas zero e um. A média de todos os padrões do conjunto correspondente a classe zero é 2 , enquanto para os padrões correspondentes à classe um é 4 .

A Fig. 3 apresenta o conjunto de dados obtido originalmente para dois padrões de conjuntos reduzidos com cada uma das quatro técnicas apresentadas, considerando uma variância de 0,5 para os padrões de ambas as classes. Pode-se verificar dos gráficos que, de uma maneira geral, as técnicas apresentadas tendem a reter os pontos localizado na fronteira entre as classes. A exceção foi a técnica SVM-KM que, como não há superposição entre as classes, tende a reter o centroide dos clusters. 

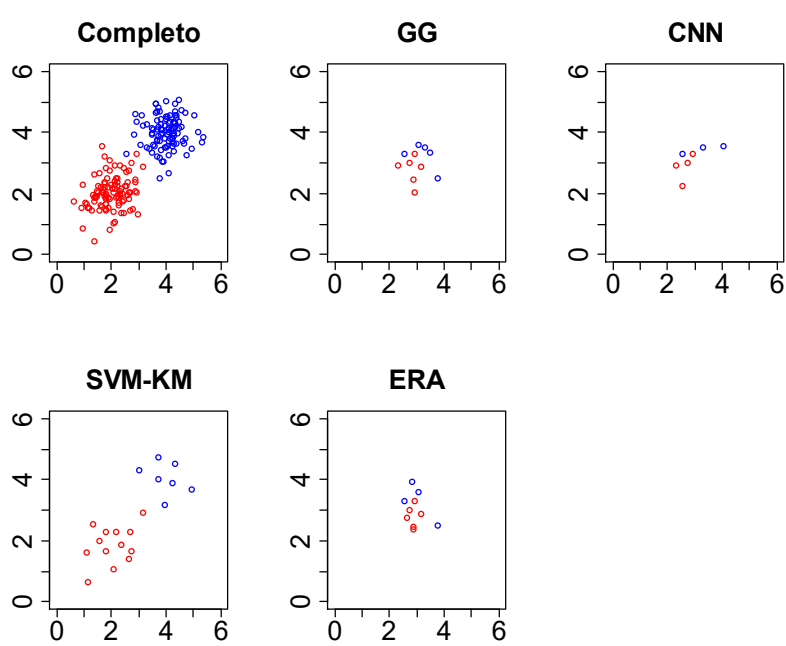

Fig. 3 Conjunto de dados original e os conjuntos reduzidos pelas técnicas propostas.

\subsection{Avaliação do efeito da superposição das classes sobre a redução}

A primeira análise deste trabalho consiste no impacto da superposição entre as classes. A variância da função de densidade de probabilidade geradora das duas classes, com emprego de dois padrões, é alterada gradativamente, conforme Fig. 4. O incremento da variância das funções geradoras implica no aumento da superposição entre as classes, o que afeta significativamente o desempenho do classificador. Espera-se que esta superposição afete também o desempenho das técnicas de redução.
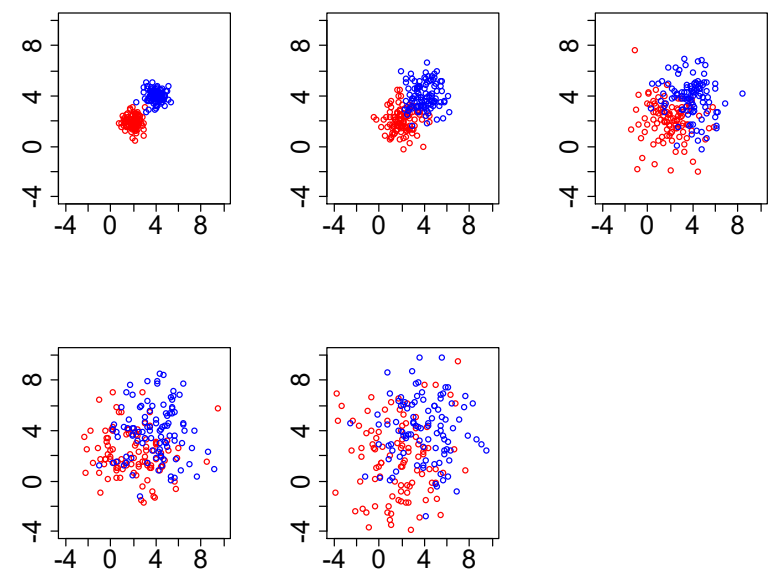

Fig. 4 Conjunto de dados obtido para diferentes valores de variância da função de densidade de probabilidade geradora $(0,5,1,1,5,2$ e 2,5$)$.

As Fig. 5 e 6 mostram, respectivamente, a média e a variância da redução obtida em valores percentuais para cada uma das metodologias testadas. Pelos gráficos apresentados nestas figuras, pode-se verificar que o aumento da superposição das classes prejudica todas as técnicas de redução. Pode-se verificar ainda que a técnica ERA é a que sofreu maior redução média em todos os casos. Em contrapartida, apresenta uma variância muito grande, o que significa que nem sempre ela levará a maior redução do conjunta de dados.

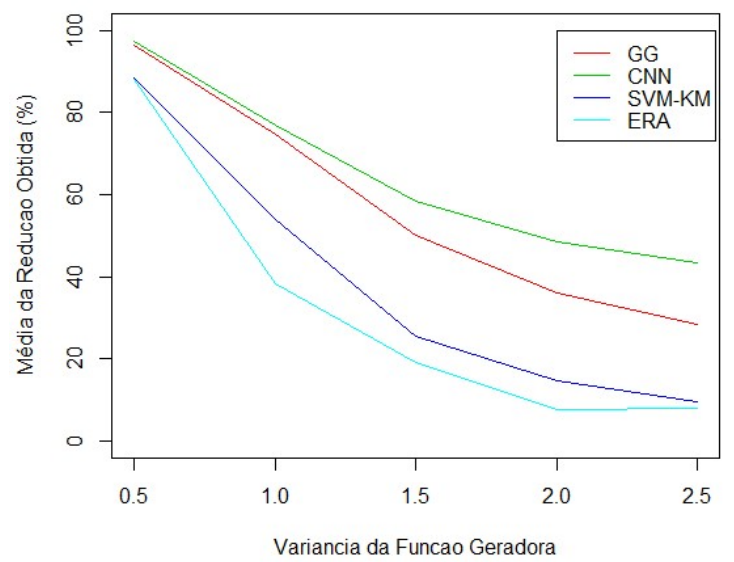

Fig. 5 Média da redução para cada uma das técnicas testada.

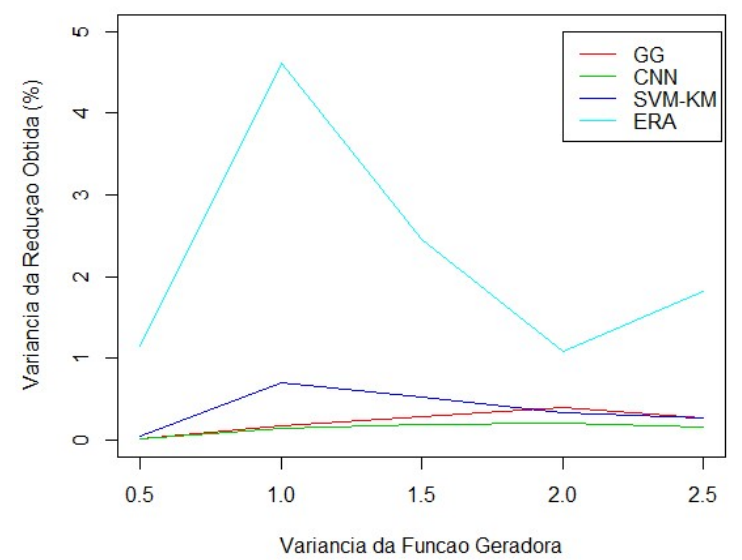

Fig. 6 Variâncias da redução para cada uma das técnicas testadas

\subsection{Avaliação do efeito da superposição das classes sobre a redução}

Além da variância da função geradora, o impacto do número de padrões é analisado. Para tal objetivo, seu valor é variado entre dois e quatro. As Fig. 7 a 10 apresentam os gráficos com as reduções médias obtidas para as quatro técnicas analisadas em função da variância da função geradora e da quantidade de padrões.

Como apresentado nas Fig. 7 a 10, a quantidade de padrões tem um impacto pequeno na redução obtida. Com o emprego do método do Grafo de Gabriel, verifica-se que o aumento no número de padrões provoca uma ligeira diminuição da redução obtida. Por outro lado, para as demais técnicas, verifica-se que um aumento na quantidade de padrões produz um ligeiro aumento na redução obtida. 


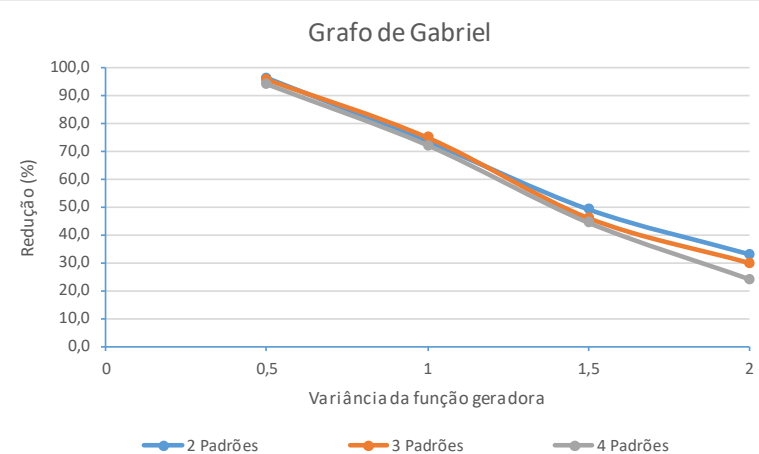

Fig. 7. Médias da redução (valor percentual) para os dados reduzidos pela técnica que utiliza o Grafo de Gabriel.

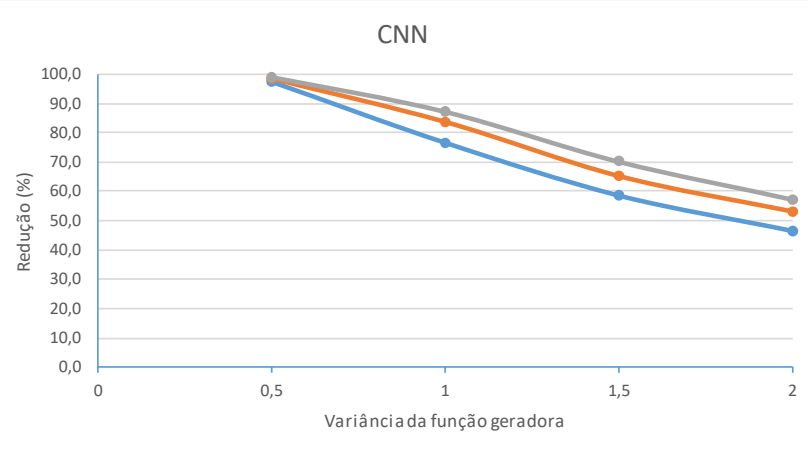

$\rightarrow 2$ Padrões $\rightarrow 3$ Padrões $\rightarrow 4$ Padrões

Fig. 8. Médias da redução (valor percentual) para os dados reduzidos pela técnica que utiliza o $\mathrm{CNN}$.

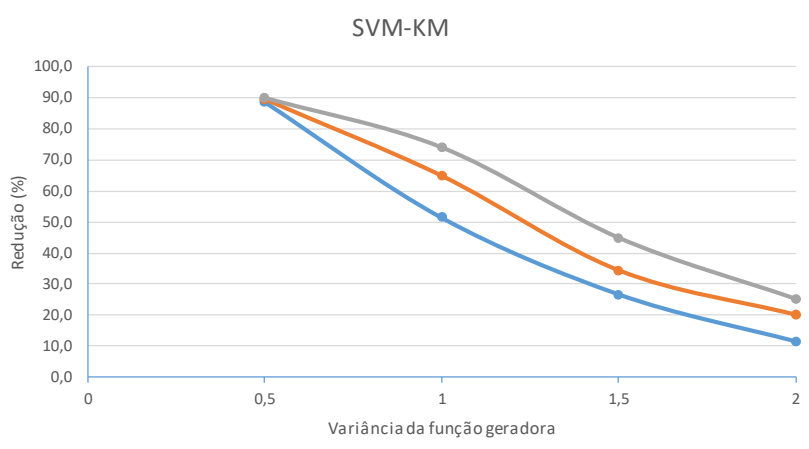

Fig. 9. Médias da redução (valor percentual) para os dados reduzidos pela técnica que utiliza o SVM-KM.

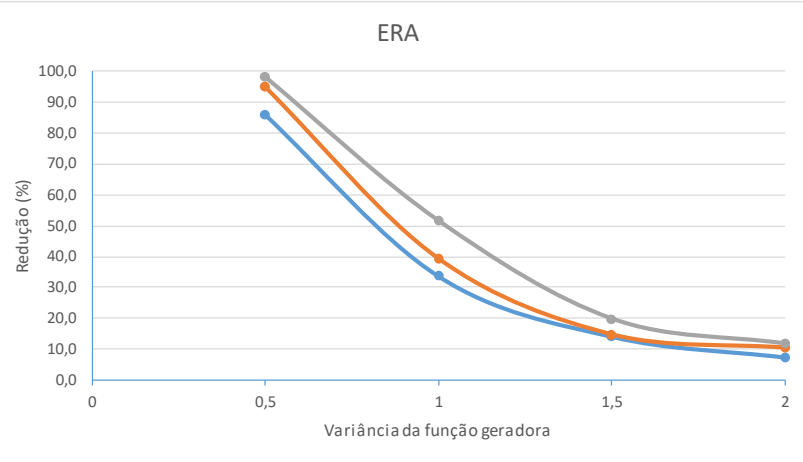

Fig. 10. Médias da redução (valor percentual) para os dados reduzidos pela técnica que utiliza o ERA.

\subsection{Avaliação do efeito da superposição das classes e do número de padrões sobre a acurácia}

Por fim, o impacto das grandezas na acurácia da RNA treinada é avaliado. As Fig. 11 a 14 apresentam os gráficos com as reduções médias da acurácia obtidas para as quatro técnicas analisadas em função da variância da função geradora e da quantidade de padrões.

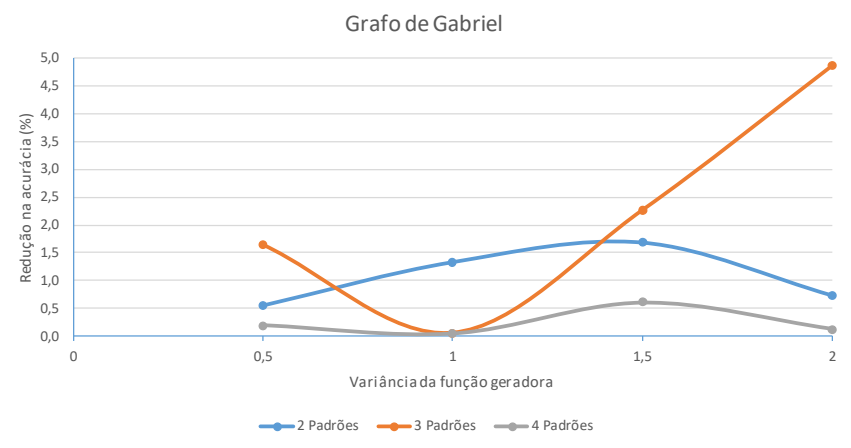

Fig. 11. Médias da redução da acurácia da RNA (valor percentual) para os dados reduzidos pela técnica que utiliza o Grafo de Gabriel.

CNN

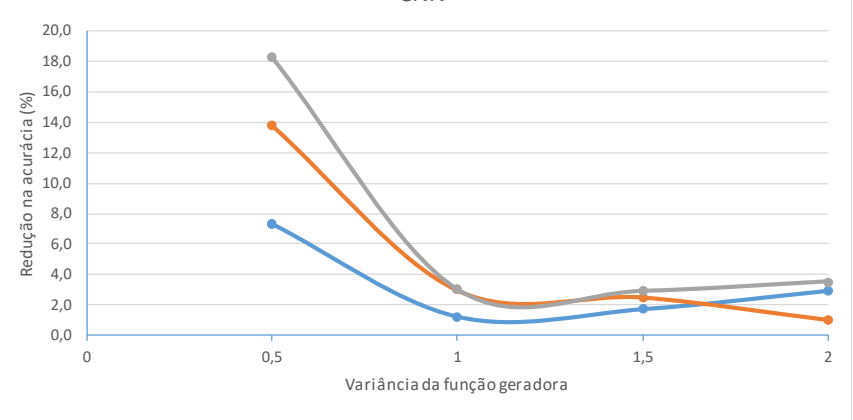

Fig. 12. Médias da redução da acurácia da RNA (valor percentual) para os dados reduzidos pela técnica que utiliza o CNN.

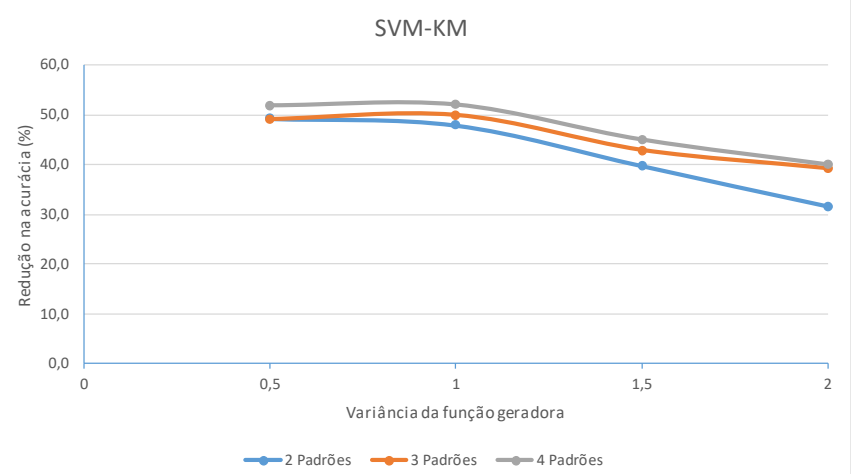

Fig. 13. Médias da redução da acurácia da RNA (valor percentual) para os dados reduzidos pela técnica que utiliza o SVM-KM.

Como apresentado nas figuras 11 a 14, as técnicas do Grafo de Gabriel e CNN provocam um impacto pouco significativo na acurácia da RNA treinada, enquanto as técnicas SVM-KM e ERA apresentam um impacto maior. Em parte, isso se deve 
ao fato destas duas técnicas serem mais conservadoras em suas reduções, com menor redução no conjunto de treinamento - vide os resultados da figura 5.

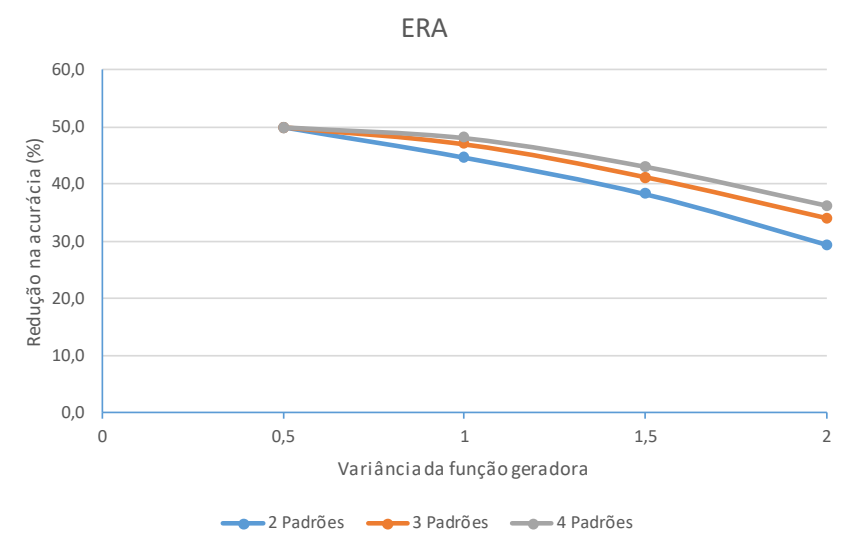

Fig. 14. Médias da redução da acurácia da RNA (valor percentual) para os dados reduzidos pela técnica que utiliza o ERA.

\section{CONCLUSÃO}

Este trabalho apresentou uma análise do desempenho de quatro diferentes metodologias que podem ser utilizadas para a redução do conjunto de treinamento de uma RNA. Das técnicas testadas, as duas que mostraram melhor desempenho foram as do Grafo de Gabriel e a $\mathrm{CNN}$, pois elas levaram às maiores taxas de redução provocando um impacto menor na acurácia da RNA. Isso ocorre porque estas técnicas são mais eficientes em detectar os pontos que estão mais próximos das interfaces entre as classes que são os pontos mais significativos para a determinação da superfície de separação.

Verificou-se, ainda, que a superposição entre as classes afeta significativamente o desempenho destas técnicas de redução. Já o efeito da quantidade de padrões, por outro lado, pode ser considerado pequeno.

Salienta-se ainda que, embora não tenha sido citado ao longo do trabalho, o consumo computacional da técnica que utiliza o Grafo de Gabriel aumenta à medida em que se aumenta também a quantidade de padrões, o que pode dificultar a sua utilização em alguns casos. A maior parte do tempo de processamento foi gasto na montagem do grafo. Assim, técnicas mais eficientes para a construção do Grafo de Gabriel ou o uso de outros grafos planares cuja construção se dê de maneira mais rápida podem ser objeto de estudos futuros.

\section{REFERÊNCIAS}

Ahmadi, M., e Ghaffari, H. (2014). Reducing the Size of Very Large Training Set for Support Vector Machine Classification, International Journal of Soft Computing and Engineering (IJSCE), 4(5): 55-61.

Barros de Almeida, M., Braga, A. P., e Braga, J. P. (2000). SVM-KM: speeding SVMs learning with a priori cluster selection and k-means. Proceedings of Sixth Brazilian Symposium on Neural Networks.
Bergmeir, C., e Benitez, J. M. (2012). Neural Networks in $R$ Using the Stuttgart Neural Network Simulator: RSNNS, Journal of Statistical Soft-ware, 46(7): 1-26.

Dalstain, T., e Kulicke, B. (1995). Neural Network-Approach to Fault Classification for High Speed Protective Relaying, IEEE Transactions on Power Delivery, 10(2): 1002-1011.

Gabriel, K. R., e Sokal, R. R. (1969). A new statistical approach to geographic variation analysis, IEEE Transactions on Power Systems, Systematic Zoology, 18: $259-278$.

Hart, P. (1968). The condensed nearest neighbor rule (Corresp.). IEEE Transactions on Information Theory, 14(3): 515-516.

Hastie, T., Tibshirani, R., e Friedman, J. (2009). The Elements of Statistical Learning - Data Mining, Inference, and Prediction. $2^{\mathrm{a}}$ ed. Springer.

Lacerda, W. S. (2006). Projeto e Implementação de Circuitos Classificadores Digitais com Contro-le da Generalização Baseado na Regra do Vizinho-maispróximo Modificada. Doutorado, Universidade Federal de Minas Gerais, Belo Ho-rizonte, Minas Gerais, Brasil.

Parez, L. G. (1994). Training an Artificial Neural Network to Discriminate Between Magnetizing Inrush and Internal Faults, IEEE Transactions on Power Delivery, 9(1): 434441.

Sidhu, T. S., Singh, H., e Sachdev, M. S. (1995). Design, implementation and testing of an artificial neural network based fault direction discriminator for protecting transmission lines, IEEE Transactions on Power Delivery, 10(2): 697-706.

Souza, J. R. S., Pereira, C. S., e De Conti, A. (2017) Designing and Training an Artificial Neural Net-work Fed by Harmonic Content of Differential Currents for Inrush Detection. International Conference on Power System Transients - IPST 2017, Seul.

Souza, J. R. S., Pereira, C. S., e De Conti, A. (2018). Reducing the Number of Tests Cases for Reliability Assessment of Protective Relays, IEEE Transactions on Power Delivery, Vol. 33(6): 3114-3121.

Zhang, W., e King, I. (2002) A Study of the Relationship Between Support Vector Machine and Gabriel Graphs. International Joint Conference Neural Networks, Honolulu. 